\section{NUCLEAR PHYSICS}

\section{Nuclear Sizes}

from our Nuclear Theory Correspondent

THE sizes of atomic nuclei are determined by the spatial distributions of their neutrons and protons. The distribution of the protons is quite well known because the electromagnetic interaction is well understood, and many studies of electron elastic scattering and of muonic $X$ rays have determined the proton distributions of many nuclei to high accuracy.

The distribution of the neutrons, on the other hand, is much less well known since this can only be found using the nuclear interaction, which is much more complicated and obscure than the electromagnetic interaction. Many different approaches have been used and although most of them tend to give neutron distributions quite similar to the proton distributions in the same nucleus the accuracy is an order of magnitude poorer.

One of the most promising of these methods depends on measuring the cross sections for the interaction of fast positive and negative pions with nuclei, and this has recently been used by a group working at the Rutherford Laboratory to obtain improved neutron distributions in a range of nuclei from carbon to lead (Allardyce et al., Nucl. Phys., A209, 1 ; 1973).

The method depends on the fact that at momenta around $1 \mathrm{GeV} / c$ negative pions interact more strongly with protons than with neutrons, whereas positive pions interact more strongly with neutrons than with protons. Since the pions are strongly absorbed they interact only with the nucleons in the nuclear surface, so the ratio of the pion reaction cross sections depends on the relative distributions of the neutrons and protons in this region. Knowing the proton distribution, information on the neutron distribution can be deduced.

In the experiment the reaction cross sections for positive and negative pions were measured at six energies for $\mathrm{C}$, $\mathrm{Al}, \mathrm{Ca}, \mathrm{Ni}, \mathrm{Sn}, \mathrm{Ho}$ and $\mathrm{Pb}$. For the first three energies the pion-nucleon cross sections differ markedly but for the remainder they are almost the same, which provides a useful check on the method. A further check is provided by including $\mathrm{C}$ and $\mathrm{Ca}$ among the targets, since for these two nuclei the neutron and proton distributions are expected to be very nearly the same.

These reaction cross sections were analysed using the relativistic optical model, which takes account of the distortion of the incident pion wave as it approaches the nucleus and its absorption as it penetrates into the interior. Since only two numbers, the positive and negative pion cross sections, are

available for each nucleus, it is not possible to determine the complete neutron density distribution. Instead, several density distributions found in previous studies of nucleon elastic scattering and of bound single-particle energies were used to calculate the expected reaction cross sections; comparison with the experimental data then showed which were the preferred distributions.

This comparison showed that the new pion experimental data favour the neutron distributions with root mean square radii within $0.1 \times 10^{-13} \mathrm{~cm}$ of the corresponding proton distributions. This is consistent with other work which indicates that for the medium weight and heavy nuclei the neutron root mean square radius is about $0.1 \times 10^{-13}$ greater

\title{
Gibbs Fracture Zone Extends Hercynian Front
}

THE Gibbs fracture zone, which crosses the present north Atlantic Ocean at about $53^{\circ} \mathrm{N}$, is apparently a transform fault which offsets the mid-Atlantic ridge axis by about $350 \mathrm{~km}$ in a roughly east-west direction. Westwards from about $35^{\circ} \mathrm{W}$ longitude to the North American continental margin, the fracture zone has been examined both bathymetrically and using seismic reflection profiling, and an intensive survey has been carried out in the area of the intersection with the mid-Atlantic ridge at about $29^{\circ} 30^{\prime} \mathrm{W}$

To the east of the ridge, on the other hand, there is little available information apart from magnetic anomalies. In Nature Physical Science next Monday (October 22), however, Cherkis, Fleming and Massingill correct this deficiency by reporting the results of a survey of the Gibbs zone between $30^{\circ} \mathrm{W}$ and $16^{\circ}$ $50^{\prime} \mathrm{W}$, and derive some significance from the fact that the zone appears to be in alignment with the Hercynian front in Europe and its extension in North America.

The new seismic profiling and bathymetric data show that the Gibbs fracture zone extends eastwards to at least $17^{\circ} \mathrm{W}$, although its characteristics change somewhat as it proceeds. The profile closest to the mid-Atlantic ridge shows that the double-trough feature persists, albeit with reduced definition, and the high central ridge is still prominent. Further east, however, the definition diminishes even further and the central ridge has either disappeared or is hidden by a dense surface reflector; there is now only a single trough, and this lies beneath an acoustically transparent sedimentary mound. Still farther east, the trough widens, sedimentary cover becoming increasingly thick; and in some ways the fracture zone comes to resemble the aseismic fracture zones of the Pacific. The zone is still observable as a buried depression at about $17^{\circ} \mathrm{W}$. than the proton root mean square radius, so that the neutrons extend slightly beyond the protons.

Subsidiary calculations confirmed that the pions interact only with the nucleons in the surface region and so the results give no information on the density distributions in the nuclear interior. Indeed even for the charge distribution most existing knowledge refers only to the surface region and little is known about the central region or the extreme fringe of low density.

It is satisfactory that the results are consistent with those already available and enhance their credibility but much still remains to be determined about the proton and neutron density distributions, particularly in the centre of the nucleus and in the outer fringe.
The Gibbs fracture zone is well aligned with the structural trends of preJurassic rocks which outcrop on the west European continental shelf and which are believed to represent the most northerly zone of Hercynian orogeny. But the Gibbs zone and the Hercynian front do not link up because the intervening Porcupine ridge and bank probably rotated to their present positions from the European continental block when the north Atlantic at $52^{\circ} \mathrm{N}$ opened, obliterating the corresponding section of the Gibbs fracture.

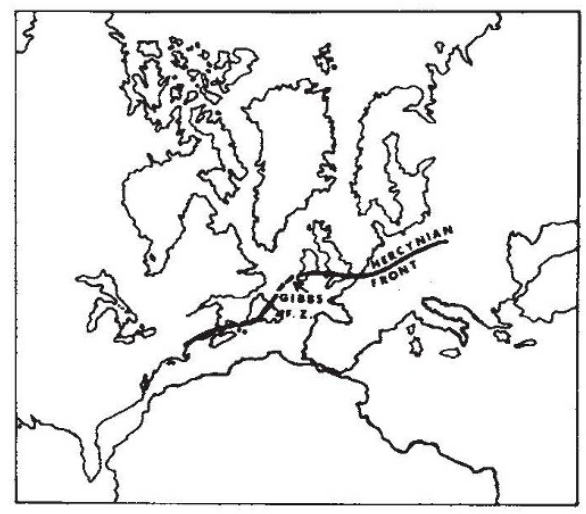

Reconstruction by Cherkis et al. of the Atlantic before the last spreading phase with positions of the Hercynian Front.

On the other side of the present Atlantic Ocean, the westward extension of the Hercynian front has been traced into North America from the western end of the Gibbs fracture by Hurley (Sci. Amer., 218, 51; 1968) and others. From the geographical and age relationships involved here, Cherkis and his colleagues conclude that the Gibbs fracture zone existed in the upper Palaeozoic as a front along which crustal plate motion occurred following the opening of the Atlantic. Later, the fracture evolved into the transform fault which now offsets the mid-Atlantic ridge. 